\title{
MINIMAL SURFACES WITH CONSTANT KÄHLER ANGLE IN COMPLEX PROJECTIVE SPACES
}

\author{
XIAOHUAN MO
}

(Communicated by Peter $\mathrm{Li}$ )

\begin{abstract}
Let $\psi: S^{2} \rightarrow C P^{n}$ be an isometric minimal immersion of the Riemann sphere $S^{2}$ into $C P^{n}$ with constant Kähler angle $\theta$. In this paper, we prove that Bolton et al.'s conjecture holds if $\theta$ is not too close to $\frac{\pi}{2}$, that is, $\psi$ is \pm holomorphic or belongs to the Veronese sequence if $|\cos \theta| \geq \frac{1}{5}$.
\end{abstract}

\section{INTRODUCTION}

Let $N$ be a Kähler manifold with the complex structure $J$ and the standard Kähler metric 〈, 〉, let $M$ be a Riemann surface; and let $\psi: M \rightarrow N$ be an isometric minimal immersion of $M$ into $N$. Then the Kähler angle $\theta$ of $\psi$, which is an invariant of the immersion $\psi$ related to $J$, is defined by $\cos (\theta)=\left\langle J e_{1}, e_{2}\right\rangle$, where $\left\{e_{1}, e_{2}\right\}$ is an orthonormal basis of $M$. The Kähler angle gives a measure of the failure of $\psi$ to be a holomorphic map. Indeed $\psi$ is holomorphic if and only if $\theta=0$ on $N$, while $\psi$ is antiholomorphic if and only if $\theta=\pi$ on $M$. In [2] Chern and Wolfson pointed out that the Kähler angle of $\psi$ plays an important role in the study of minimal surfaces in $N$. From this point of view, we would like to know all isometric minimal immersions of the constants Kähler angle in $N$.

In this paper, we shall discuss this problem when $N$ is a complex projective space with the Fubini-Study metric of constant holomorphic sectional curvature 4, denoted by $C P^{n}$. Let $S^{2}(K)$ be a 2-dimensional sphere of constant Gaussian curvature $K$. Examples of minimal surfaces of constant Kähler angle in $C P^{n}$ are given in [1] and [3]. For example, for each integer $p$ with $0 \leq p \leq n$, there exist full isometric minimal immersions $\psi_{n, p}: S^{2}\left(K_{n, p}\right) \rightarrow C P^{n}$, where $K_{n, p}=4 \backslash[n+2 p(n-2)]$. Each $\psi_{n, p}$ possesses holomorphic rigidity; that is, two such immersions differ by a holomorphic isometry of $C P^{n}$.

Characterizing minimal surfaces of constant Kähler angle in $C P^{n}$, Bolton et al. [1] conjectured that, if the Kähler angle of an isometric minimal immersion $\psi: S^{2} \rightarrow C P^{n}$ is constant, then the Gaussian curvature of $\psi$ is also constant, when the immersion is neither holomorphic, antiholomorphic, nor totally real. They gave an affirmative answer to this conjective for $n \leq 4$. We would like

Received by the editors July 27, 1992 and, in revised form, September 28, 1992.

1991 Mathematics Subject Classification. Primary 53A10; Secondary 49Q05, 53C42, 53C55.

This work was supported by the Postdoctor Foundation in China. 
to discuss this conjecture under some additional conditions. We prove the following

Main Theorem. Let $C P^{n}$ be an n-dimensional complex projective space of constant holomorphic sectional curvature 4 and let $S^{2}$ be a topological 2-sphere. Let $\psi: S^{2} \rightarrow C P^{n}$ be a linearly full isometric minimal immersion with constant Kähler angle $\theta$. Then $\phi$ is \pm holomorphic or belongs to the Veronese sequence $\psi_{n, p}$ if $|\cos \theta| \geq \frac{1}{5}$.

\section{CONJECTURE OF KäHLER ANGLE}

Let $\psi: S^{2} \rightarrow C P^{n}$ be a linear full isometric minimal immersion. Let $\psi_{0}, \ldots, \psi_{n}$ be the harmonic sequence determined by $\psi$ with $\psi=\psi_{q}$ for some $q=0, \ldots, n$. Let $L_{0}, \ldots, L_{n}$ be its associated sequence of complex line subbundles of the trivial bundle $S^{2} \times C^{n+1}$. If

$$
\partial_{p}: T^{(1,0)} S^{2} \otimes L_{p} \rightarrow L_{p+1}, \quad p=0, \ldots, n-1,
$$

are the associated bundle holomorphisms described on [1, p. 602] then the ramification index $r\left(\partial_{p}\right)$ of $\partial_{p}$ is a nonnegative integer. Let $\gamma_{p}$ denote the type $(1,0)$ energy density of $\psi_{p}$. Then the Kähler angle of $\psi_{q}$, denoted by $\theta_{q}$, satisfies

We set

$$
t_{p}=\left(\tan \left(\theta_{q} / 2\right)\right)^{2}=\gamma_{p-1} / \gamma_{p}
$$

$$
\delta_{q}=\frac{1}{2 \pi i} \int_{S^{2}} \gamma_{q} d \bar{z} \wedge d z>0
$$

for $q=0, \ldots, n-1$. Here $z$ is a local complex coordinate on $S^{2}$.

Suppose $t_{q}$ constant.

Lemma 1. If $t_{q} \leq p /(p+1)$ for some $p \in\{1, \ldots, n\}$, then $q \leq p-1$.

Proof. By the hypothesis, $(p+1) \gamma_{q-1} / p \leq \gamma_{q}$. From [1, Proof of Lemma 9.4, p. 619]

$$
\frac{1}{2 \pi i}\left(\delta_{q}-\frac{p+1}{p} \delta_{q-1}\right) \int_{S^{2}} \gamma_{q-1} d \bar{z} \wedge d z \geq 0 .
$$

From this we can conclude that $p \delta_{q}-(p+1) \delta_{q-1} \geq 0$.

Suppose that $q \geq p \geq 1$. Then, from [1, (3,24)], it follows that

$$
\begin{aligned}
-p \delta_{q}+(p+1) \delta_{q-1}= & (p+1) q+(n-q)(q-p) \\
& +\frac{(n-q+1+p)}{n+1} \sum_{k=0}^{q-2}(k+1) r\left(\partial_{k}\right) \\
& -\frac{p}{n+1}(n-q) q r\left(\partial_{q-1}\right)+\frac{q-p}{n+1} \sum_{k=q}^{n-1}(n-k) r\left(\partial_{k}\right) \\
& +\frac{(p+1) q(n-q+1)}{n+1} r\left(\partial_{q-1}\right) \\
> & \frac{(p+1+n-q) q}{n+1} r\left(\partial_{q-1}\right) \geq 0 .
\end{aligned}
$$

Hence $p \delta_{q}-(p+1) \delta_{q-1}<0$ which contradicts the estimate above. Hence $q \leq p-1$. 
Lemma 2. If $t_{q} \geq(p+1) / p$ for some $p \in\{1, \ldots, n\}$, then $q \geq n-p+1$. Proof. The proof is similar to the proof of Lemma 1.

Lemma 3. $|\cos \theta| \geq \frac{1}{5}$ if and only if either $t_{q} \leq \frac{2}{3}$ or $t_{q} \geq \frac{3}{2}$.

Proof. Trig identities prove Lemma 3.

Theorem. If $|\cos \theta| \geq \frac{1}{5}$, then $\psi$ is part of the Veronese sequence, holomorphic, or antiholomorphic.

Proof. By Lemma 3, $t_{q} \leq \frac{2}{3}$ or $t_{q} \geq \frac{3}{2}$, say the former holds. Then the hypothesis of Lemma 1 holds for $p=2$, and therefore $q \leq 1$. If $q=0$, then $\psi$ is holomorphic. If $q=1$, then $t_{0}=0$ and $t_{1}$ are both constant, so $\psi$ is part of the Veronese sequence by [1, Remark 5.5, p. 612]. Similarly, if $t_{q} \geq \frac{3}{2}$, then $q \geq n-2+1=n-1$, so either $q=n-1$ or $q=n$. Again either $\psi$ is antiholomorphic or $\psi$ is part of the Veronese sequence by [1, Remark 5.5, p. 612].

\section{REFERENCES}

1. John Bolton, Gary R. Jensen, Marco Rigoli, and Lyndon M. Woodward, On conformal minimal immersions of $S^{2}$ into $C P^{n}$, Math. Ann. 279 (1988), 599-620.

2. S. S. Chern and J. G. Wolfson, Minimal surfaces by moving frame, Amer. J. Math. 105 (1983), 59-83.

3. S. Bando and Y. Ohnita, Minimal 2-spheres with constant curvature in $P_{n}(C)$, J. Math. Soc. Japan 39 (1987), 477-487.

Institute of Mathematics, Fudan University, Shanghai 200433, China

Current address: Department of Mathematics, Peking University, Beijing, 100871, China 